\title{
11 Ein Beitrag aus dem Politmagazin REPORT MAINZ
}

Vom Spielfilm sei nun, wie angekündigt, der große Sprung gemacht: Betrachtet sei im Folgenden ein Beitrag aus einer Sendung von REPORT MAINz, einem seit über 50 Jahren bestehenden Politmagazin der ARD. Auch ein journalistisches Fernsehformat wie dieses zeigt das Phänomen filmischen Metaphorisierens. Bei solchen Magazinbeiträgen ist eine permanente Sprachpräsenz die Regel (anders als bei Spielfilmen, die diesbezüglich ja eine extrem große Variabilität aufweisen), häufig in Form eines stetigen Wechsels von einem Voice-over und Originaltönen. ${ }^{1}$ Dennoch sind Fernsehmagazine nicht bebilderte Texte: Genauso wie bei Spielfilmen sind sprachliche Äußerungen in ihrem Artikulationskontext der audiovisuellen Komposition zu betrachten. Auch hier hat man es mit einer audiovisuellen Kommunikation zu tun, in der Verstehensprozesse, Prozesse der Bedeutungsgenerierung, über eine reflexive, sich in der Zeit entfaltende Wahrnehmung modelliert werden.

Der im Folgenden analysierte, etwa sechseinhalbminütige Beitrag, ${ }^{2}$ der am 20. Oktober 2008 ausgestrahlt wurde (Autoren: Steffen Hudemann, Thomas Reutter, Gottlob Schober), hat die damalige globale Banken- und Finanzkrise als zeitgeschichtlichen Kontext und widmet sich dem Thema des Verlierens von Bankmanagern in einer solchen Krise. ${ }^{3}$ Makroanalytisch betrachtet, ist bei diesem Magazinbeitrag ein Polarisieren die dominante dynamische Verlaufsform des Metaphorisierens. Ein Innehalten sowie ein pointiertes Rahmen sind zwei weitere makroanalytisch auffällige Formen, die im Folgenden rekonstruiert werden. Beobachtbar ist, dass die metaphorischen Themen, die metaphorischen Erfahrungsbereiche, die man durch andere erfährt, hier jeweils recht klar sprachlich artikuliert werden - Spielfilme sind da, wie gesehen, subtiler bzw. variabler. Anders im Vergleich zu diesen ist beim Magazinbeitrag zudem, dass das Aktivieren der Metaphorizität von Sprachäußerungen ein zentraler Bestandteil des Metaphorisierens ist.

Das Magazinintro ist vorbei, und nach einer knappen Begrüßung der $\mathrm{Zu}$ schauer beginnt der Moderator der Sendung, Fritz Frey, sogleich mit der Anmoderation des Beitrags (00:10-00:53). Im Zuge der rund 40 Sekunden, die er jetzt

1 Diesen Wechsel haben sie etwa mit Nachrichtenfilmen gemein. Vgl. für Metaphernanalysen von Fernsehnachrichten Müller/Kappelhoff: Cinematic Metaphor.

2 Die Angabe des Zeitumfangs inkludiert die An- und Abmoderation.

3 Die vorliegende Analyse des Magazinbeitrags baut auf einer gemeinsam mit Hermann Kappelhoff, Cornelia Müller, Susanne Tag und Sarah Greifenstein durchgeführten Analysearbeit hierzu auf. Vgl. Müller/Schmitt: Audio-Visual Metaphors of the Financial Crisis sowie auch Müller/ Kappelhoff: Cinematic Metaphor.

Ә Open Access. ( 2020 Christina Schmitt, publiziert von De Gruyter. @B BY-Nc-ND Dieses Werk ist lizenziert unter der Creative Commons Attribution-NonCommercial-NoDerivatives 4.0 Lizenz. https://doi.org/10.1515/9783110614619-011 
spricht, nähert sich ihm zunächst die Kamera in recht zügigem Tempo mit einer seitlichen Fahrt, sodass man zu Beginn noch das Setting des Fernsehstudios sieht: mit einem Tisch, hinter dem der Moderator sitzt, und einem Monitor daneben. Mit wenigen stimmlich und gestisch pointiert intonierten Worten, und zusammen mit der Einblendung des ikonischen Bildes des damaligen (zu diesem Zeitpunkt schon mehrere Jahre) mächtigsten Mannes der Deutschen Bank - Josef Ackermann: mit breitem Lächeln und zum Victoryzeichen geformter erhobener Hand, fotografiert im Gerichtssaal am ersten Verhandlungstag eines Prozesses gegen ihn wegen Untreue -, wird die Schuld der Bankmanager an der Finanzkrise skizziert genauso wie ihre gesellschaftliche Verurteilung als amoralische Akteure:

\footnotetext{
Moderator

„In Zeiten der Finanzkrise steht eine ganze Branche am Pranger. Die Linke, populistisch wie eh und je, wünscht sich Bankmanager gar ins Gefängnis. Das ist natürlich Blödsinn. Richtig ist, dass der Millionenverzicht des Herrn Ackermann ,zugunsten verdienter Mitarbeiter ein weiteres Indiz für den Realitätsverlust einer völlig abgehobenen Geldkaste ist.“
}

Nun zoomt die Kamera immer weiter an den Moderator heran, in die fernsehtypische Einstellung des talking head, und im Fokus ist so jetzt allein das Gesagte:

\section{Moderator}

„Da mag sich der ein oder andere trösten, dass so mancher Banker nun den Chefsessel räumen muss. Doch nicht zu früh gefreut: Viele der gefeuerten Banker werden, wenn sie fallen, weich fallen. Steffen Hudemann und Gottlob Schober zeigen, wie es geht.“

Wie man sich verlierende Bankmanager vorzustellen hat, rückt die Anmoderation mit ihrer audiovisuellen Inszenierung und sprachlichen Rhetorik dadurch als Thema des folgenden redaktionellen Beitrags ins Zentrum. Und sie tut dies metaphorisierend, indem verlierende in Opposition zu gewinnenden Bankmanagern entworfen werden; ein Kontrast, der über eine vertikale Achse aufgemacht wird: Die abgehobene Geldkaste kontrastiert mit dem Fallen gefeuerter Chefs einem Fallen, das noch als weiches Fallen spezifiziert wird.

Ein Metaphorisieren nimmt hier seinen Anfang, das sich mit einer komplexen Elaborierung über den gesamten Beitrag im Verlauf von sechs Szenen entfaltet. Im diskursiven Ereignis des Filme-Sehens, wie es die cinematic communication dieser audiovisuellen Fernsehbilder evoziert, emergiert das Verständnis, dass ein Gefühl des Verlierens und Scheiterns in der Bankenkrise ein scharfer Kontrast ist $\mathrm{zu}$ einem Gefühl des Gewinnens und Erfolgreich-Seins - und dass ein solches Kontrast-Gefühl nicht für Bankmanager in Anspruch zu nehmen ist.

Die Schärfe des Kontrasts evozieren horizontal wie vertikal zu beschreibende Dynamiken des Metaphorisierens. Denn zum einen vollzieht der Report in seinem 
zeitlichen Verlauf (horizontale Ebene) durch das Zusammenspiel von sprachlichen Äußerungen und audiovisueller Inszenierung ein ständiges Wechseln von Erfahrungen des Gewinnens als Obensein und des Verlierens als Untensein, ein ständiges Wechseln also zwischen beiden Polen der in der Anmoderation sprachlich artikulierten vertikalen Achse. Und zugleich vollzieht sich synchron mit diesem Wechseln zwischen Oben und Unten ein Wechseln zwischen weiteren gegensätzlichen Erfahrungsqualitäten (vertikale Ebene) - und zwar ausschließlich evoziert durch die audiovisuelle Inszenierung, durch filmische Expressivität. Gewinnen in der Finanzkrise ist dadurch nicht nur als Abgehoben- und Obensein erfahrbar, sondern zugleich als ein Sein in einem geschlossenen Innen und als fokussierte, unbeeinträchtigte Vorwärtsbewegung. Verlieren in der Finanzkrise hingegen wird nicht nur als ein Fallen, eine Abwärtsbewegung, ein Untensein erlebt, sondern zugleich als ausgeschlossenes Außen, als Langsamkeit, als ein Unterbinden von Bewegung. (Für eine Makroübersicht zu zentralen Metaphorisierungsaktivitäten vgl. Abb. 35.)

Einer exemplarischen mikroanalytischen Rekonstruktion dieser Dynamiken des Metaphorisierens sei eine weitere makroanalytische Betrachtung vorangestellt. Denn weshalb ist das sich so entfaltende Empfinden eines Verlierens, das in scharfem Kontrast zum Gefühl des Gewinnens emergiert, nicht für Banker, die ihren Managerposten verlieren, in Anschlag zu bringen?

Bemerkenswerterweise tritt die Akteursgruppe der Bankmanager während des Beitrags überhaupt nicht direkt in Erscheinung: weder als Gewinner, noch als Verlierer. Und das, obwohl ja die Anmoderation zum Beitrag auf eben deren Verlieren in der Finanzkrise zusteuert - „Viele der gefeuerten Banker werden, wenn sie fallen, weich fallen. Steffen Hudemann und Gottlob Schober zeigen, wie es geht.“ Vordergründig jedoch inszeniert der Beitrag ganz andere Akteure: So werden privatwirtschaftliche Jobvermittler von gefeuerten Bankern, sogenannte Outplacement-Berater, als Gewinner der Bankenkrise etabliert (Szene 1, 3 und 6). Als Verlierer der Bankenkrise hingegen werden Kleinanleger, die ihr Erspartes verloren haben (Szene 2 und 3), prominent zur Anschauung gebracht ebenso wie einfache Bankmitarbeiter, die ihre Anstellung aufgrund von Stellenstreichungen verlieren (mit einem Betriebsratstatement, Szene 4). Zwischen diesen Gruppen wechselt der Beitrag hin und her; mit Blick auf sie entfaltet sich eine audiovisuelle Wahrnehmung des scharfen Kontrasts zwischen Gewinnen und Verlieren.

Bankmanager hingegen treten - den „Realitätsverlust der völlig abgehobenen Geldkaste“ in spezifischer Weise aktivierend - in dieser audiovisuellen Inszenierung nur indirekt, körperlos, abstrakt in Erscheinung: indirekt, wenn ein verbal-gestisches Statement des Geschäftsführers der Firma von Rundstedt Human Resources Partners gescheiterte Bankmanager, die „nicht gesehen werden“ wollen, als Outplacement-Geschäftsfeld skizziert (Szene 1); gesichts- und körper- 
Ein wirkliches Gefühl des Verlierens ist ein scharfer Kontrast

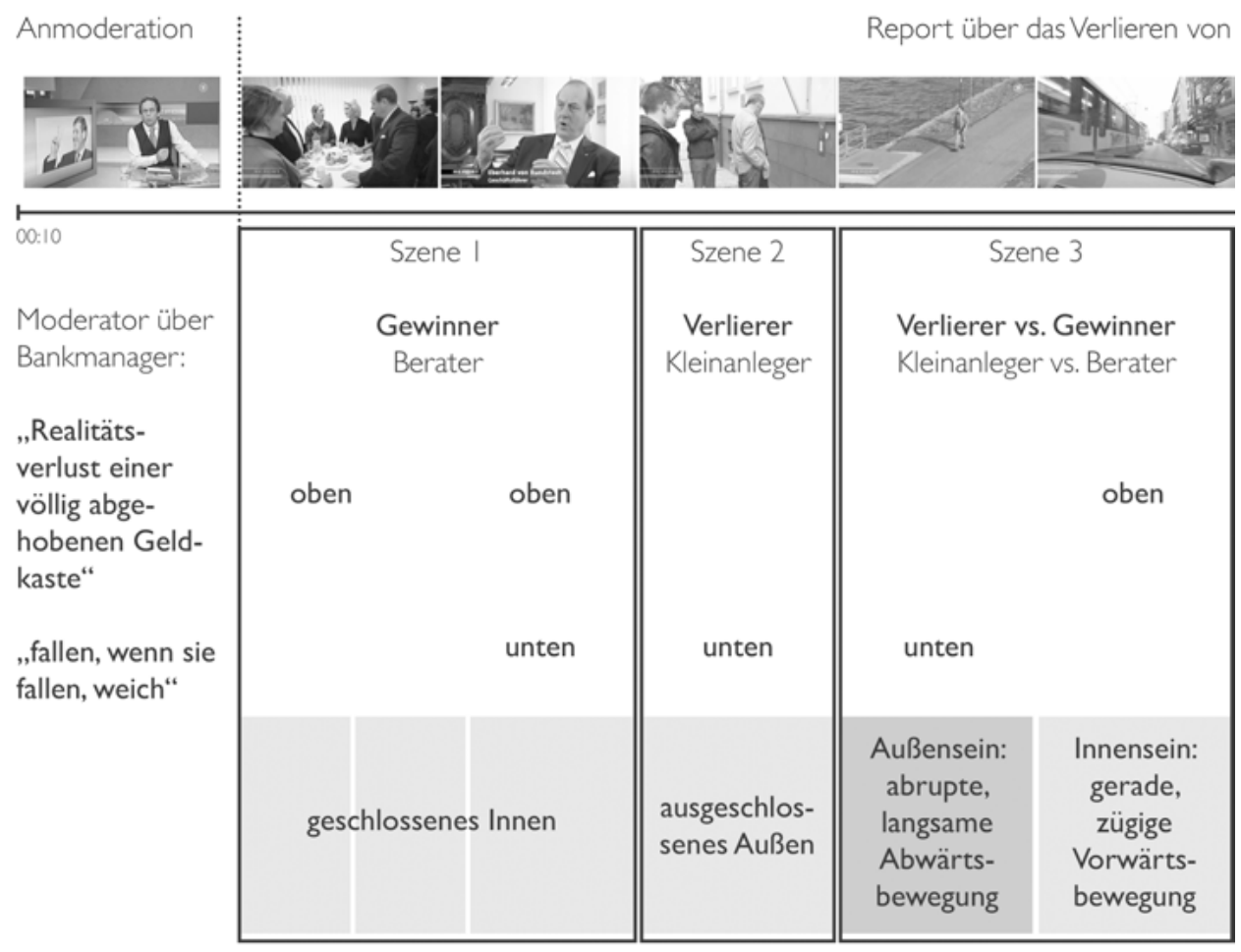

Abb. 35: Übersicht zentraler Metaphorisierungsaktivitäten im REPORT MAINz-Beitrag zum Verlieren von sprachlichen Äußerungen und audiovisueller Inszenierung. 
zu einem Gefühl des Gewinnens

Bankmanagern in der Finanzkrise

\section{Abmoderation}

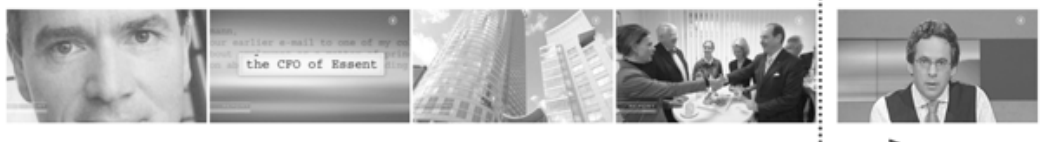

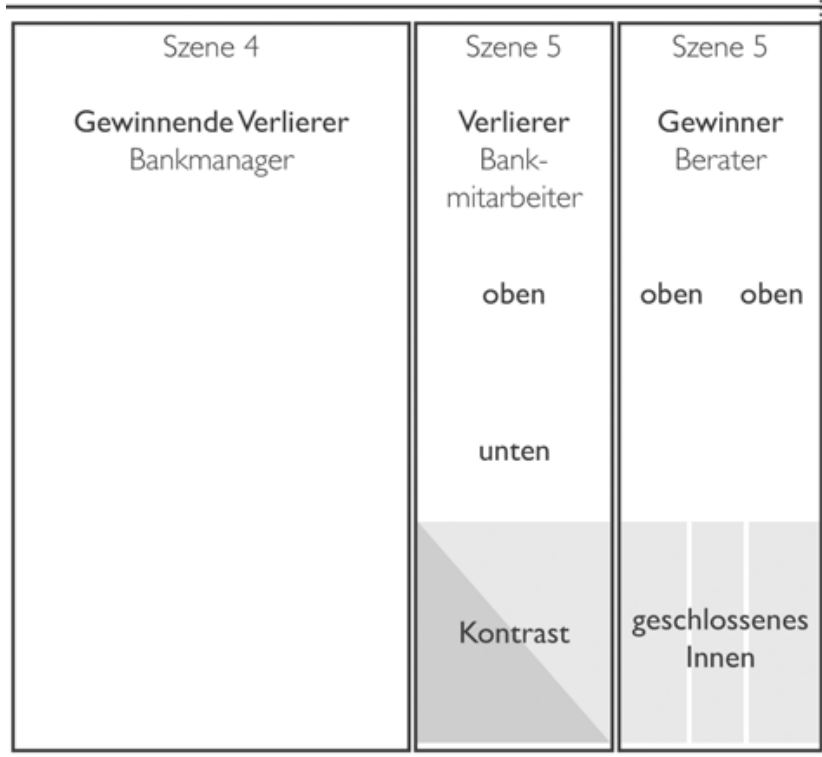

Bankmanagern in der Finanzkrise (20.10.2008), evoziert durch das Zusammenspiel von 
los, wenn lediglich eine verzerrte Stimme aus der Gegensprechanlage einer krisengeschüttelten Bank zu Kleinanlegern vor verschlossener Tür dringt (Szene 2); abstrakt, wenn die einzige Fallbeispiel-Szene ihren Höhepunkt im Hineinzoomen in ein (Bewerbungs-)Foto eines geschassten Bankers findet, überblendend in einen grafisch abstrakten Hintergrund mit dem Ausdruck eines Rechercheemailwechsels, der von der erneuten Finanzvorstandsposition des Managers zeugt (Szene 4). Damit einhergehend emergiert der Eindruck eines Realitätsverlusts der Banker gerade darin, dass das Scheitern der Bankmanager nicht (oder nicht als Fokus) in dem Wahrnehmungsparcours sich sprachlich wie audiovisuell entfaltender Erfahrungsbereiche verhandelt wird, mit welchem die Realität des Verlierens als scharfer Kontrast zum Gewinnen entworfen wird: Die Banker sind eben dieser Wirklichkeit - wie sie nun ausführlich für die ersten drei Szenen und skizzenhaft für den Beitragsabschluss rekonstruiert sei - enthoben.

\section{Gewinnen und Verlieren verstehen - und Outplacement}

Erste Szene, 00:52-02:16

Auf eine zügige, immer wieder ansetzende Vorwärtsbewegung folgt das Moment einer Desorientierung, die sich in Neuorientierung auflöst, bis sich zuletzt Ruhe ausbreitet. Auf eine Montagebewegung von der Halbtotalen bis zur Großaufnahme folgt eine Montage von Nah- und Großaufnahmen, bis zuletzt eine lange Einstellung auf einem einzelnen schweren Körper ruht. Auf Häppchen, Sekt und feiernde Berater folgt eine Erläuterung ihres Outplacement-Unternehmens, bis zuletzt ihr Chef ein Statement zu gescheiterten Bankmanagern abgibt.

Drei Ausdrucksbewegungen fügen sich hier zu einer szenischen Komposition, die als Ganzes eine spezifische Wahrnehmungsbewegung vollzieht (wie unten ausführlich beschrieben werden wird): Reflexiv nimmt man im Zuschauen ein Bewusstsein wahr, das in Etappen in ein geschlossenes Innen gelangt. Dieses geschlossene Innen zeigt sich als Erfahrungsbereich des Gewinnens. Und es geht einher mit Elaborierungen der vertikalen Achse, wie sie die Anmoderation zum Beitrag aufgemacht hatte. Mit der Passage in dieses geschlossene Innen ereignet sich in der Szenenmitte zugleich eine analytisch interessante Verschiebung: Sind Gewinnen und Verlieren am Anfang und am Ende der Szene die Themen im Metaphorisieren, so werden sie dort, um es mit Cameron zu sagen, zu Vehikeln, zum Wahrnehmungserleben, mit dem ein Verstehen von Outplacement evoziert wird.

Die Szene beginnt mit einer Elaborierung des Erfahrungsbereichs des Gewinnens (erste Szene, erste Ausdrucksbewegungseinheit, 00:53-01:21, Abb. 36), der hier mit wirtschaftlichem Erfolg exemplifiziert wird. Berater der Firma von Rund- 
stedt Human Resources Partners werden als „Gewinner der Bankenkrise“ vorgestellt, beim „Sektempfang in der Niederlassung direkt an der Düsseldorfer Kö“: Man sieht eine Gruppe von Männern und Frauen in Businesskleidung mit glänzenden Sektgläsern, und von „glänzend“ laufenden Geschäften ist im Voice-over die Rede. Und wiederholt sind Aufwärtsbewegungen in Szene gesetzt: Das Eingießen von Sekt (der selbst noch ein Symbol für das Feiern von Erfolg hier ist) wird in Großaufnahme gezeigt, und eine Kamerabewegung nach unten betont das schäumende Aufsteigen der Perlen - ebenso wie ein Zoom-in das gemeinsame Erheben der Gläser intensiviert, wenn die Berater anstoßen. Mit einer Montage dreier Großaufnahmen verlagert sich die Aufwärtsbewegung dann in die Mimik, in lachende Gesichter.

Doch inwiefern hebt mit diesem Beitragsauftakt zugleich eine Wahrnehmungsbewegung an, die (im Verlauf der gesamten ersten Szene) ins Zentrum eines geschlossenen Innens gelangt? Hierfür sind Montagebewegung und Bildkomposition in den Blick zu nehmen. In einer Halbtotalen sind die Berater zu sehen, in einem geschlossenen Kreis gruppiert um runde Stehtische, die Körper sind auf das Innere, das Zentrum dieser Kreisformationen ausgerichtet. Die Kamera nähert sich einer dieser geschlossenen, runden Formation, bleibt ihr zunächst noch außen vor - eine Großaufnahme vom gefüllt werdenden Sektglas unterbricht das Annähern. Doch dann ist der Kamerablick mit einer neuen Kadrierung bereits Teil der Kreisformation und dringt mit dem Zoom, der das Sekt-Anstoßen begleitet, sogleich noch weiter vor. Er ist jetzt Teil dieser geschlossenen Gruppe, ist mit den Großaufnahmen zum Schluss auf Augenhöhe mit den Beratern - das Voice-over endet, und der Originalton beginnt mit einem von drei glücklich lächelnden Beratern:

„Sie sehen uns hier gerade ein Gläschen trinken. Wir haben heute Morgen einen Ex-Banker verabschiedet in seinen neuen Job.“

Mit dieser direkten Ansprache des Interviewers durch einen der Berater schreibt man die Bewegung des zügigen Vordringens zu dem geschlossenen Innen den Journalisten $\mathrm{zu}^{4}$ - doch es ist zuvorderst eine reflexive Wahrnehmungsbewegung, die sich in jenem Akt des Filme-Sehens realisiert.

4 Diese wurden als zeigende Instanz ja auch von der Anmoderation hervorgehoben. 

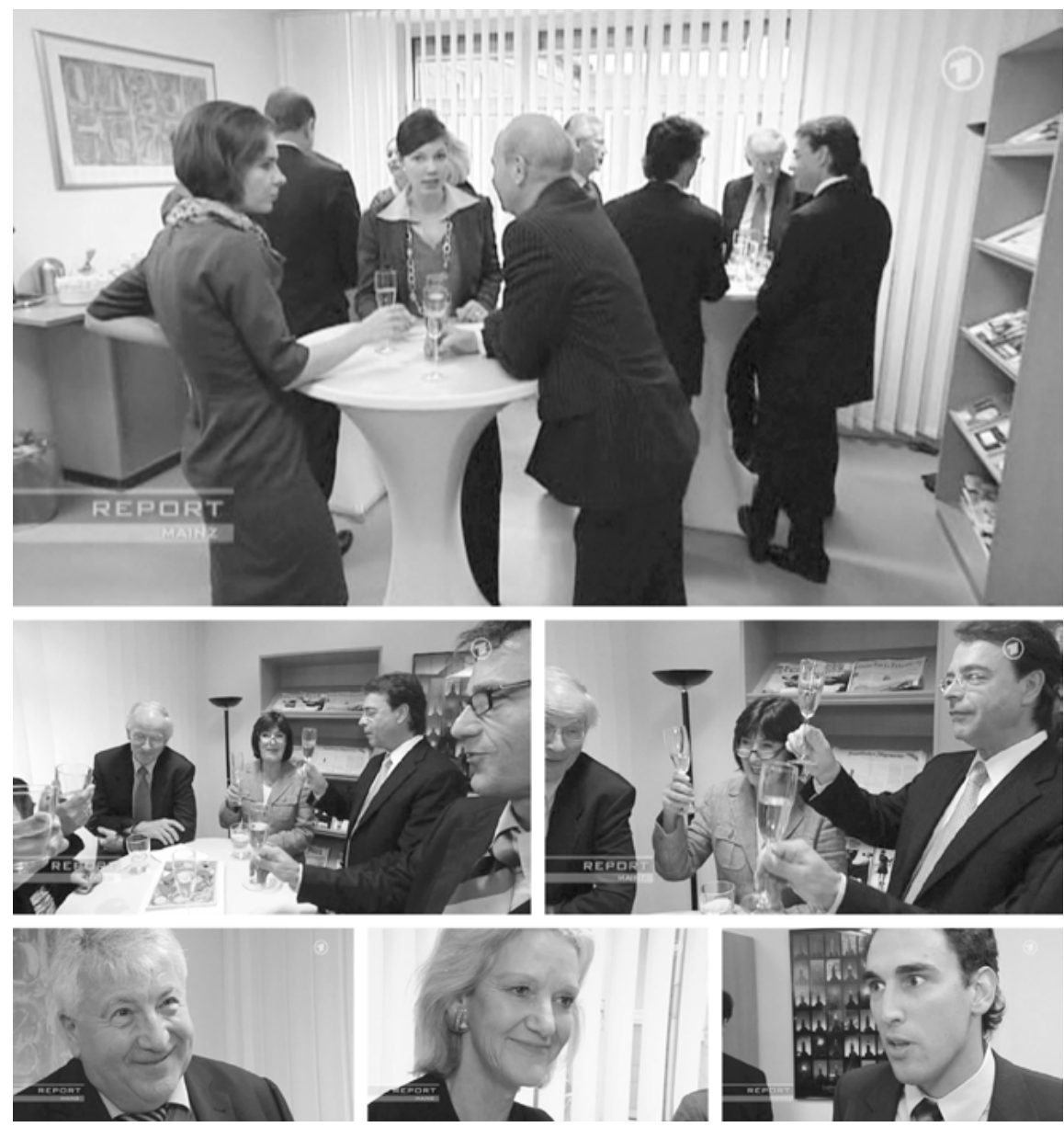

Abb. 36: Eine Montage von der Totalen bis hin zur Großaufnahme artikuliert eine zügige, immer wieder ansetzende Vorwärtsbewegung in ein geschlossenes Innen. (REPORT MAINZ-Beitrag zur Bankenkrise, erste Szene, erste Ausdrucksbewegungseinheit, 00:53-01:21.)

War eben, im Anfang der Szene, noch das Gewinnen im Fokus, verlagert dieser sich jetzt sprachlich zu dem Geschäftsfeld, mit dem das Beratungsunternehmen wirtschaftlich erfolgreich ist:

Voice-over

„Outplacement bezeichnet eine Beratung zur beruflichen Neuorientierung, die der alte Arbeitgeber bezahlt. Also eine Beratungsbegleitung vom Ausscheiden aus dem alten Unternehmen bis zum Wiedereintritt in ein neues Unternehmen.“ 
Im Metaphorisieren setzt damit ein Innehalten ein (erste Szene, zweite Ausdrucksbewegungseinheit, 01:21-01:39, Abb. 37). Nicht mehr Gewinnen (oder Verlieren) in der Bankenkrise steht im Fokus, sondern Outplacement wird erörtert. Die filmische Expressivität, die audiovisuelle Geste, mit deren Vollzug obige sprachliche Äußerung realisiert wird, lässt für diesen neuen Fokus hier in der Szenenmitte nun Verlieren und Gewinnen selbst zum Vehikel werden.

Während das Voice-over von der „Beratung zur beruflichen Neuorientierung“ spricht, fokussiert die Kamera Kommunikationsakte: sprechende Münder, zuhörende Gesichter, gestikulierende Hände, Blickachsen. Ganz nah rückt die Kamera dabei an die Körper, Gesichter und Hände heran. Ganz nah gleitet sie über Körper, die sich so überlagern und fragmentieren. Anders als noch zuvor, ist jetzt keine Übersicht, kein Überblick mehr über eine Situation feiernder Berater gegeben. Vielmehr evoziert diese Inszenierung sowohl eine extreme Körperlichkeit und Nähe als auch ein Gefühl des Nicht-mehr-platziert-Seins (displacement), ein Gefühl der Dynamisierung und Desorientierung. Es ist ein Gefühl, das sich wandelt: Entfaltet die Montage der Körperfragmente zunächst ein Erleben von Nichtmehr-platziert-Sein, von Haltlosigkeit, findet dann ein Rhythmus Eingang in dieses Bild.

Ein Rhythmus, der subtil einen neuen, ordnenden Aspekt in diese Montage der fragmentierten Nahaufnahmen bringt. Ein Rhythmus, den vier audiovisuelle Synchronisationspunkte gestalten, wenn Kopf-, Hand- und Kamerabewegungen zum Schluss mit der Intonation der vom Voice-over gesprochenen Worte getaktet sind, mit denen die Begriffserörterung abgeschlossen wird: „vom Ausscheiden aus dem alten Unternehmen bis zum Wiedereintritt in ein neues Unternehmen“. 5

Das Wahrnehmungserleben der Ausdrucksbewegung vollzieht so einen Wechsel vom Verlust zum Wiedergewinn von Orientierung und Halt. Im Zusammenpiel von Sprachäußerung und audiovisueller Geste artikuliert sich damit ein subtiles körperliches Verstehen von den im Outplacement-Geschäftsfeld wirkenden Prozessen: Ein Gefühl von out-/dis-placement transformiert sich mit einem Rhythmus in ein Gefühl von re-placement.

5 Für Synchronisationspunkte im audiovisuellen Fluss vgl. Chion: Audio-Vision. Auch im Hornbach-Spot, der hier im vorliegenden Kapitel abschließend analysiert werden wird, spielen solche Synchronisationspunkte eine zentrale und zugleich ganz andere Rolle. 

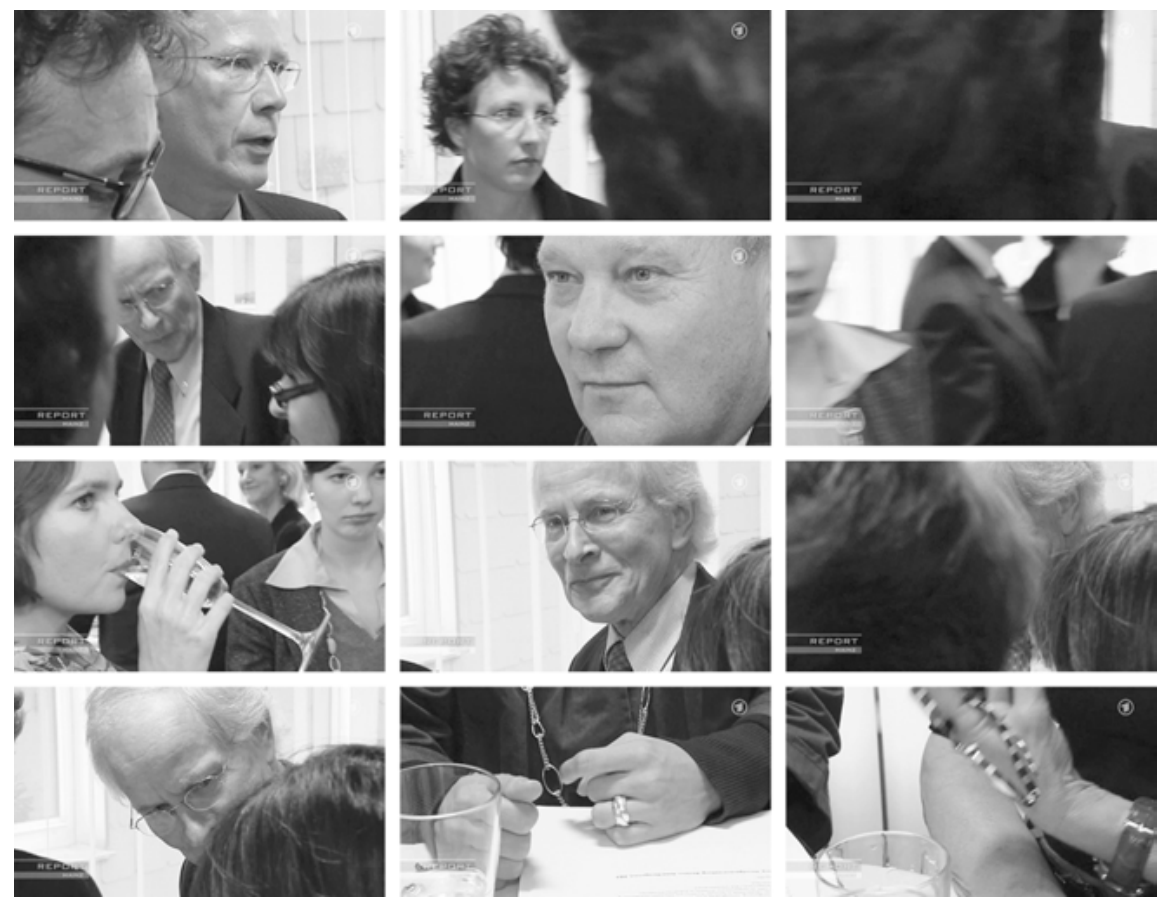

Abb. 37: Eine Montage von Kameraschwenks in Groß- und Detailaufnahme artikuliert einen Orientierungsverlust, erst audiovisuelle Synchronisationspunkte evozieren zum Schluss einen Rhythmus und damit einen Wiedergewinn von Orientierung (REPORT MAINz-Beitrag zur Bankenkrise, erste Szene, zweite Ausdrucksbewegungseinheit, 01:21-01:39).

Und eben dieses Erleben, das die filmische Expressivität hier entfaltet, ist die Passage der reflexiven Wahrnehmungsbewegung in das geschlossene Innen der Gewinner, der Berater. Diese hatte in der vorangegangenen Ausdrucksbewegung in dessen Peripherie begonnen. Und im nun Folgenden gelangt sie zuletzt in das Zentrum dieses geschlossenen Innens: zum Geschäftsführer.

Hierfür baut sich zunächst eine ironisch-distanzierende Haltung auf, und Gewinnen und Verlieren rücken wieder in den metaphorischen Fokus. Und beides - Aufbau der Haltung und Fokusverschiebung - hängen zusammen. Denn die Sequenz, mit der die erste Szene abschließt (dritte Ausdrucksbewegung, 01:39-02:16, Abb. 38), beginnt mit einer Montage, bei dem zu Hörendes und zu Sehendes einander widersprechen, bei der vom Verlieren die Rede ist, aber Gewinnen gezeigt wird. So sagt das Voice-over, bezugnehmend auf die vorherige Erklärung von Outplacement als einer „Beratung zur beruflichen Neuorientierung“: 
„In Zeiten der Krise ein optimales Instrument für gescheiterte Bankmanager. Das findet der Star der Branche, Eberhard von Rundstedt.“

Doch zur Rede von den „Zeiten der Krise“ sieht man Kanapees in Großaufnahme. Und während die Worte sich den „gescheiterten Bankmanagern“ zuwenden, nähert sich der Blick der geschlossenen Kreisformation feiernder Berater.

Erst jetzt isoliert eine Großaufnahme den „Star der Branche“ von den anderen Beratern. Und mit einem Umschnitt vom Foyer des Sektempfangs zum Büro des Geschäftsführers gelangt die Wahrnehmungsbewegung ins Innere des geschlossenen Innens der Gewinner. Der Eindruck, im Zentrum eines Innens zu sein, das das Außen ausschließt, wird hier nicht zuletzt über den Sound evoziert. In der gesamten bisherigen Szene unterlegte ein akustischer Teppich aus dichtem Stimmengewirr und entspannter Piano-Bar-Musik jedes Voice-over wie auch die Originalton-Statements mit ihrem trockenen Klang. Hier jedoch ist der Klangraum nun leer: Ihn füllt allein die sonore, leicht hallende Stimme des Geschäftsführers. ${ }^{6}$ Und mit der akustischen Ruhe geht die Regungslosigkeit der zuvor durchgängig sehr dynamischen Kamera einher: Mit einer langen, leicht untersichtigen Einstellung ruht die Kamera auf dem schweren, massiven Körper des sitzenden von Rundstedt und dem schweren Holzmöbel. Das in der filmischen Expressivität gründende Gefühl eines geschlossenen Innens, mit dem der Star der Krisengewinner hier zur Wahrnehmung kommt, ${ }^{7}$ ist nun der Hintergrund für ein Wahrnehmungserleben von Gewinner- und Verlierersein als zweier Pole auf einer vertikalen Achse. Der audiovisuell sinnlichen Qualität der Aufwärtsbewegung, mit der die Berater zum Szenenanfang als Gewinner wahrnehmbar waren, wird jetzt die Gegenbewegung gegeben. So erläutert der Geschäftsführer die These, dass eine Outplacement-Beratung für in der Finanzkrise gescheiterte Bankmanager optimal ist, mit folgenden Worten:

„Das Wichtigste ist, dass diese Menschen, die ja hoch, von hoch oben gestürzt sind, gestern noch gefeiert, heute meiden die Leute womöglich den Kontakt mit ihnen, dass die wieder Mut fassen und dass man ihnen Techniken und Ansätze vermittelt. Das können die alles. Nur sie wollen es nicht, sie wollen eigentlich wegtauchen und nicht gesehen werden. Und wir sagen ihnen, genau das ist falsch. Jeder macht mal Fehler.“

6 Zur hallenden Stimme im Film vgl. Rick Altman: Sound Space. In: Sound Theory, Sound Practice. Hrsg. v. Rick Altman. New York: Routledge 1992, S. 46-64.

7 Dass ein solches situativ entstehendes, spezifisch artikuliertes Gefühl eines geschlossenen Innens etwas grundlegend Anderes meint als einfach ein repräsentatives Verständnis von einem Zimmer als einem Innenraum, und auch etwas anderes als das Container-Image-Schema, sollte deutlich geworden sein. 

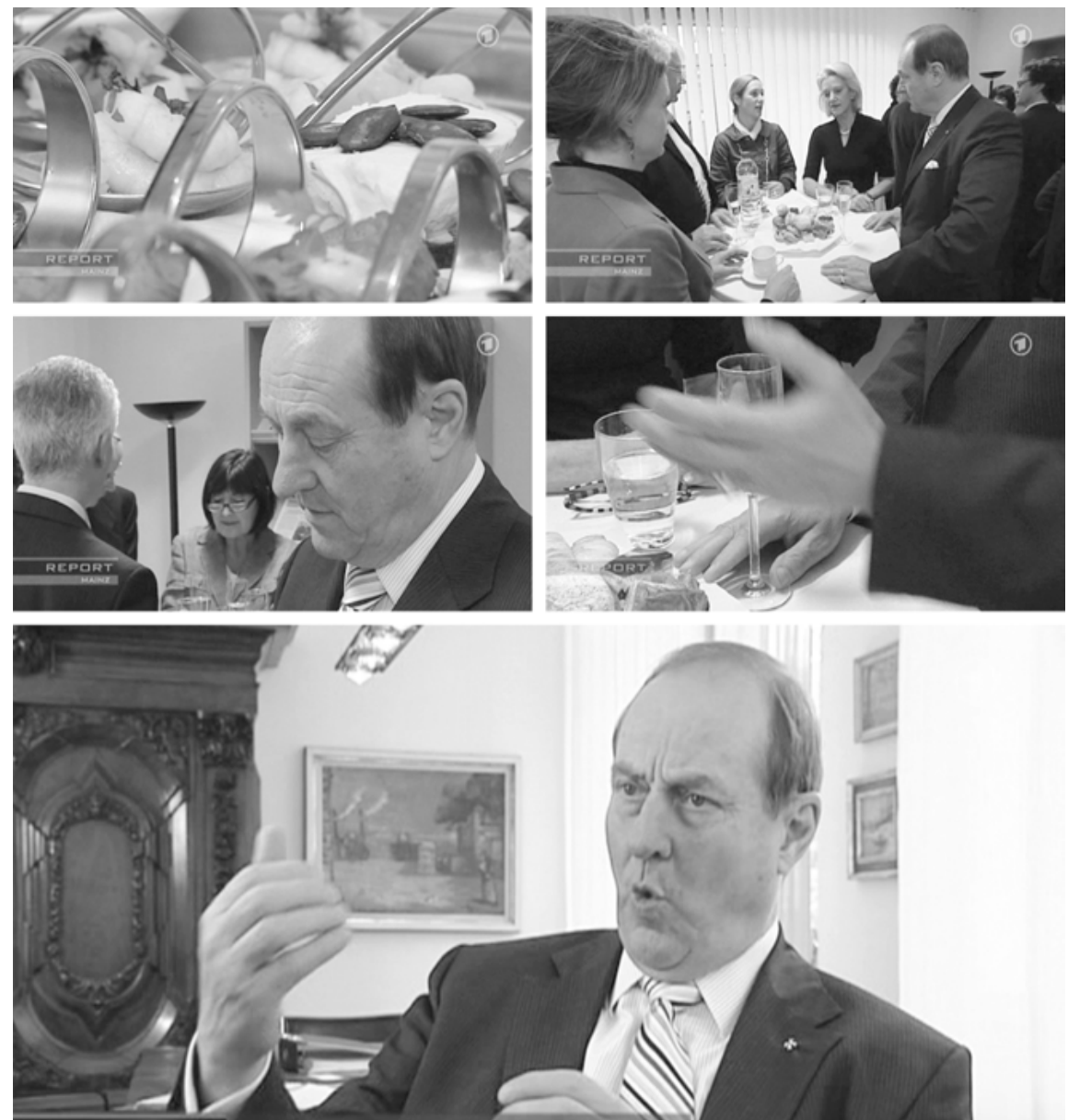

REPERT

MAINZ
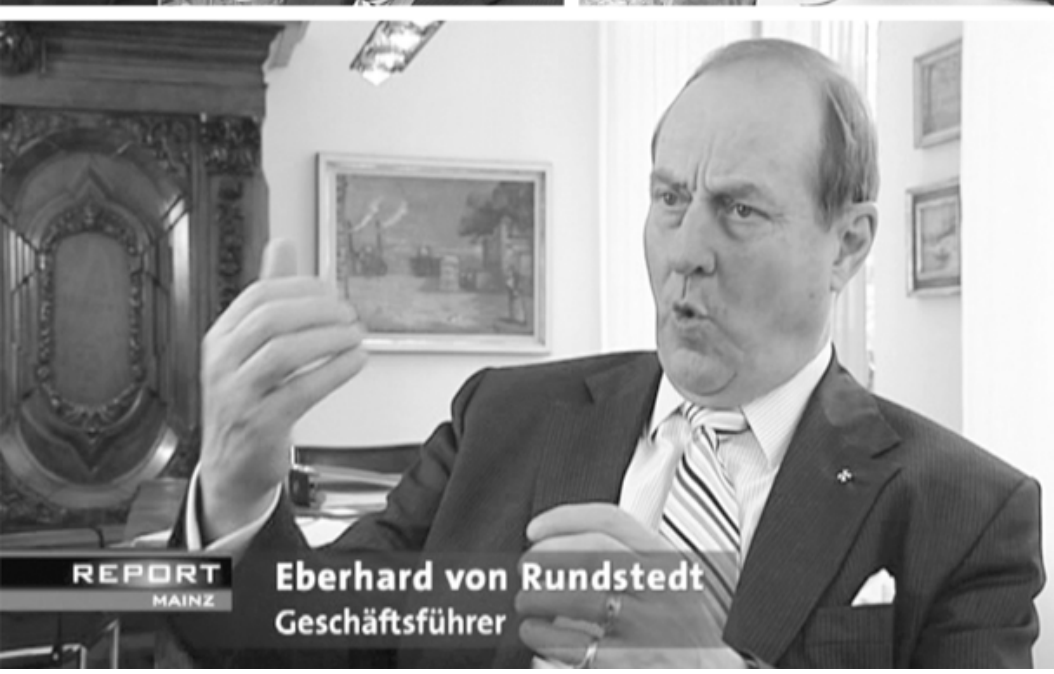

Abb. 38: Eine vorherige audiovisuelle Dynamik kommt im Zentrum eines geschlossenen Innens zur Ruhe - mit einer lang andauernden statischen Einstellung und einem akustisch entleerten, hallenden Klangraum (REPORT MAINZ-Beitrag zur Bankenkrise, erste Szene, dritte Ausdrucksbewegungseinheit, 01:39-02:16.)

Sein Sprechen über die gescheiterten Manager ist hier integral verbunden mit einem ständigen Gestikulieren (das durch die audiovisuelle Inszenierung bereits vor dem Umschnitt in die Einzelsituation ins Zentrum der Aufmerksamkeit gerückt wird, wenn die Großaufnahme, die den Geschäftsführer aus der Gruppe isolierend heraushebt, vom Gesicht hinunter zu den Händen gleitet, die zu gestikulieren beginnen): Und zusammen mit „hoch, von hoch oben gestürzt“ voll- 
zieht von Rundstedt mit seiner rechten Hand eine klar konturierte Abwärtsbewegung.

Doch ist der Kontext des verbal-gestischen Abwärts, dieser sinnlichen Qualität, die audiovisuell prominent zur Anschauung gebracht ist, hier eben das durch die filmische Expressivität evozierte Gefühl der Gewinner: das Innen, das das Außen auschließt. Noch im Scheitern gehört man als Banker zu den Gewinnern. Krise heißt hier: Sekt und Kanapees. Wenn man fällt, fällt man weich. Wirkliches Verlieren ist etwas anderes, wirkliches Verlieren existiert nur im scharfen Kontrast zum Gewinnen: Eben dies modelliert sich im weiteren Verlauf des Beitrags.

\section{Verlieren und Scheitern verstehen}

Zweite Szene, 02:16-02:54

Eine Bewegung kommt in Gang, fokussiert und anhaltend nach vorne drängend und gerät ins Stocken, kommt nicht weiter: Eine Kamerabewegung hebt an und vollzieht sich mit kontinuierlicher, dynamischer Qualität in einer lang andauernden Einstellung, die über weite Strecke mit einer zentralperspektivischen Bildkomposition gestaltet ist - und dann bricht eine Montage kurzer Einstellungen die Kontinuität ab, entzieht der Bewegung ihre vorandrängende Kraft. Was mit einer solchen filmischen Ausdrucksbewegung hier in dieser zweiten Szene zur Wahrnehmung kommt, ist die Zielgerichtetheit der Bewegung einer Gruppe von Männern auf der Straße - und ihr Zum-Stehen-Kommen vor einer Hauswand. ${ }^{8}$ (Vgl. Abb. 39.)

Die Kamera scheint von Anfang an Teil dieser Gruppe, agiert in Nahaufnahmen aus ihr heraus. Die Männer, bepackt mit Rucksäcken und Outdoorkleidung, die Blicke nach unten gerichtet, gehen los und die Kamera folgt ihnen - und mit einem lang andauernden Gang, bildkompositorisch in einer engen, tunnelhaften Häuserflucht, bewegt sich diese Gruppe, dieses bewegliche Außen, zielgerichet und zügig auf eine Wand, auf die Grenze zu einem Innen zu: Die sich zuletzt nach unten ausrichtende kontinuierliche Kamerabewegung endet in der Massivität der Nahaufnahme einer Videotürsprechanlage, eingelassen in einer grauen Hausmauer. Es folgt eine Schuss-Gegenschuss-Montage, die in Großaufnahmen versteinerter Gesichter mündet: Aufnahmen des leblosen Videoeauges aus kaltem Metall in der harten Mauer wechseln mit Aufnahmen nach unten geneigter Köpfe, regungslos im Gesichtausdruck. Realisiert wird ein Gefühl des Ausgeschlossenseins, eines ausgeschlossenen Außens.

8 Die zweite Szene besteht aus einer einzelnen Ausdrucksbewegung. 

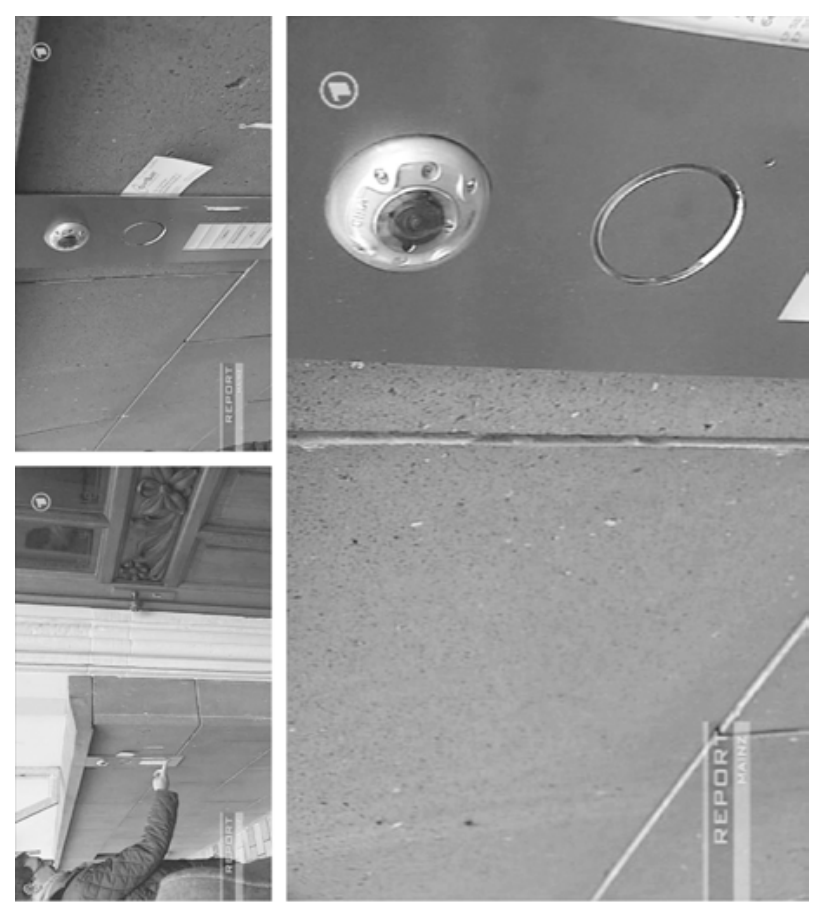

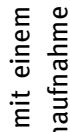

ह $\frac{\pi}{\frac{\pi}{2}}$

范.

离 듬

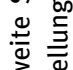

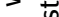

¿ั

:

导 禀

히

홍

흘

กำ

空苋

on 0

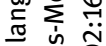

जิ

壳 돈

of $\frac{1}{d}$.

5 on d

沓造

ए ज

उे जे कू

D

ข
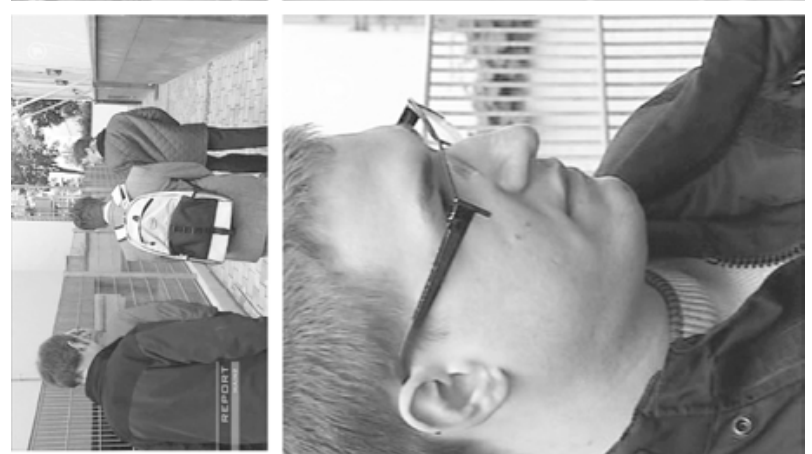

这

品 $\frac{\bar{c}}{\mathrm{~d}}$

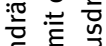

僖主

등 品

ว

कo :

号

志 离

$\frac{\pi}{n} \tilde{n}$

资 $\subseteq$

흥 등

证

艺 苛

$\stackrel{\breve{v}}{\breve{y}}$

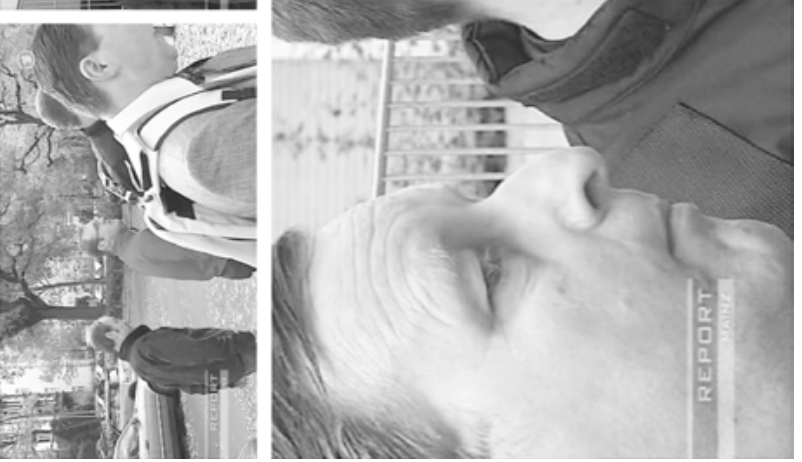

ह का

紊

국

든 군

.๘

西

言 衣 के 뜸 \% 호 赔鬲

‥ 음

ब̈ं है 음

ㅇํ음 
Und dieses sich in der filmischen Expressivität, durch Kamerabewegung, Bildkomposition und Montage artikulierende Gefühl des Ausgeschlossenseins ist zugleich durchwirkt mit dem sinnlichen Erleben eines rein visuell inszenierten Nach-Untens. Denn mit dem zielgerichteten Gang, der an der Mauer zum Halt kommt, und auch mit der dann folgenden Schuss-Gegenschuss-Montage richtet sich alles nach unten: die Körperhaltung, die gesenkten Köpfe und Blicke der Männer wie auch der Kamerablick.

In der Wahrnehmung dieser durch eine Ausdrucksbewegung gestalteten Szene, die eine Gruppe von Kleinanlegern vor der Frankfurter Filiale der isländischen Kaupthing-Bank zeigt, setzt sich so der zuvor begonnene Prozess des Metaphorisierens fort, mit dem Verlieren und Scheitern als ein Gefühl emergiert, das klar mit dem Gewinnen und Erfolgreich-Sein kontrastiert. Denn im Erleben eines ausgeschlossenen Außens und Sich-nach-unten-Ausrichtens, wie es die audiovisuelle Geste evoziert, entfaltet sich audiovisuell-sprachlich der Erfahrungsbereich des Verlierens und Scheiterns: Zuvorderst auf einer semantischen Ebene findet ein Elaborieren dieses Erfahrungsbereichs statt, wenn mit dem Voice-over während der lang andauernden Einstellung von der Verzweiflung der Sparer und ihrer Furcht um ihr Geld ob eingefrorener Konten die Rede ist. Performativ entfaltet sich der Erfahrungsbereich, wenn die Bitte der Kleinanleger, Auskunft von der Bank zu bekommen (eine kontinuierliche Bewegung entfaltetet sich zielgerichtet ...), scheitert (... und kommt einer Montage kurzer Einstellungen zum Erliegen). Denn als Originalton ist bei der Schuss-Gegenschuss-Montage der versteinerten Gesichter (der regungslosen Gesichter der Kleinanleger und der Großaufnahme des Videoauges in der Steinmauer) folgender Dialog zu hören:

\footnotetext{
Kleinanleger

„Wichtig ist uns natürlich einfach nur zu wissen, dass alles Menschenmögliche versucht wird, dass wir unser sauer erspartes Geld wieder irgendwann bekommen können.“

Verzerrte Stimme aus der Gegensprechanalage

„Ganz genau. Und genau das ist auch der Fall. Es wird alles Menschenmögliche getan.“
}

Die verzerrte Stimme aus der Gegensprechanlage erstickt den von den Sparern gesuchten Dialog, wenn sie letztlich nur ein Echo der an sie gerichteten Worte äußert. Und mit ihrer steinernen, leblosen, keiner sichtbaren, menschlichen Körperlichkeit zugeordneten Erscheinungsweise führt die Stimme die Rede vom Menschenmöglichen ad absurdum. Scheitern und Verlieren wird hier evident. 


\section{Verlieren im scharfen Kontrast mit Gewinnen verstehen \\ Dritte Szene, 02:54-03:52}

Mit Beratern einerseits und Kleinanlegern andererseits haben die ersten beiden Szenen zwei Personengruppen eingeführt: Krisengewinner und Krisenverlierer. Die daran anschließende, mittlere Szene des Beitrags konfroniert nun sehr konzentriert einzelne Vertreter dieser beiden Gruppen miteinander: Der kurzen Schilderung eines Kleinanlegerschicksals folgt ein Kommentar des Outplacment-Geschäftsführers zur Flüchtigkeit von Skandalen. Über die szenische Komposition zweier Ausdrucksbewegungen spitzt sich hierbei das Kontrastgefühl von Verlierersein und Gewinnersein zu.

Die Szene beginnt mit einer Ausdrucksbewegung, in deren Zuge ein Verständnis von Verlieren als eine Abwärtsbewegung und Langsamkeit im Außen evoziert wird (dritte Szene, erste Ausdrucksbewegungseinheit, 02:54-03:23, vgl. Abb. 40, obere Reihe). Ein Kleinanleger geht am Fluß, am Main entlang. Eine Nahaufnahme mit leichter Untersicht zeigt ihn größer als die hinter ihm aufragende Frankfurter Bankenskyline, die zusammen mit dem Fluss bildkompositorisch eine Horizontale betont, durch Figuren- und Kamerabewegung mit einer Orientierung von rechts nach links. Das Voice-over spricht von 100.000 Euro - und jetzt wechselt die Einstellung, während der Satz weitergeführt wird:

Voice-over

„100.000 Euro hat er als Festgeld bei der Kaupthing Bank angelegt, eigentlich bombensicher.“

Unvermittelt ist der Kleinanleger jetzt von großer Entfernung aus einer Totalen in schräger Vogelperspektive zu sehen. Und nicht mehr die Horizontale, sondern die Schräge bildkompositorisch betont. Links oben im zweigeteilten Bildfeld: eine Fläche aus Wasser und rechts unten die steinerne Fläche der Uferpromenade. Mit dem Einstellungswechsel dauert der langsame Gang des Kleinanlegers am Wasser zwar weiter an. Doch ist diese Bewegung jetzt abrupt abwärts gerichtet, von rechts oben nach links unten.

Die Einstellung wechselt ein weiteres Mal. Das Bild kehrt von der Schrägen wieder in die Horizontale zurück, und der Kleinanleger steht nun still, platziert in der linken Bildfeldhälfte. Wie zu Anfang ist er in Nahaufnahme zu sehen - doch die Bankentürme überragen und fixieren ihn jetzt, anders als noch zu Beginn der Montagesequenz:

\section{Kleinanleger}

„Und den Vorwurf muss ich mir jetzt gefallen lassen, dass ich die Ausbildungsversicherung meiner vier Kinder verzockt habe. Und ich habe nicht gezockt. Ich habe nur gespart.“ 
Durch eine solche Inszenierung des Kleinanlegers realisiert sich über den Verlauf der Montage der drei Einstellungen eine filmische Expressivität, die eine abrupte, nach links orientierte Abwärtsbewegung artikuliert. In ebendiesem Ausdruck einer Abwärtsbewegung vollzieht sich die Wahrnehmung des unvorhergesehenen Verlusts von sicher geglaubtem Geld und Respekt. Und mit der farblichen Gestaltung der Bildkomposition und den Kameraperspektiven scheint zudem der Umraum, das Außen, den Kleinanleger immer mehr zu absorbieren - der Kleinanleger wird zum integralen Teil eines Außens.

Die filmische Expressivität, die den weiteren Szenenverlauf ausgestaltet, vollzieht die Gegenbewegung zu einer solchen Wahrnehmung. Jetzt ist erneut von Rundstedt im Fokus. Und anküpfend an den Beitragsbeginn, der den Geschäftsführer als Star der Outplacement-Berater einführte, wird, mit einer Autofahrt, für diesen Krisengewinner mit der jetzigen Ausdrucksbewegung erneut ein Oben in einem geschlossenen Innen inszeniert - das sich nun jedoch zugleich mit dem Erleben einer zügigen, nach rechts-vorne gerichteten flüssigen Bewegung verbindet (dritte Szene, zweite Ausdrucksbewegungseinheit, 02:54-03:23, vgl. Abb. 40, untere Reihe).

Der Rückspiegel eines Autos zeigt das unbewegliche Gesicht des Geschäftsführers, das fokussiert geradeaus schaut. Es ist das Bild eines angeschnittenen Gesichts, das ins Abstrakte geht, eines statischen, gerahmten Innens in Nahaufnahme, das sich durch eine städtische Straßenlandschaft bewegt. Mit dem Einstellungswechsel setzt sich die zügige Bewegung dessen flüssig fort. Jetzt jedoch macht eine Subjektive, ein point of view shot das fokussierte Blicken selbst sichtbar: In großer Tiefe, eng und steil laufen die Achsen von Häusern am Straßenrand, Asphalt und einer Straßenbahn aufeinander $\mathrm{zu}$ - eine extreme Fluchpunktperspektive dominiert die Bildkomposition dieses Blicks auf eine Straße aus einem fahrenden Auto heraus. Das gerade und fokussierte Blicken im Vorwärts - ein flüssiges und ununterbrochenes, ungestörtes Bewegen - setzt sich mit dem nächsten Einstellungswechsel auf einer horizontalen Achse nach rechts fort. ${ }^{9}$ Die untersichtige Perspektive der lang andauernden statischen Nahaufnahme zeigt den fahrenden Berater erhöht, und erst jetzt ist das fokussierte Blicken in einen sichtbaren menschlichen Körper integriert - in einen massiven Körper, der bildkompositorisch zugleich mit seinem Umraum, dem Innen des Autos verschmilzt: Organisch fügt sich das Gesicht mit dem Beige der Leder-Innenverkleidung. Ein zügiges Bewegen eines unbewegten Innens in einem bewegten Außen realisiert sich hier.

9 Letztlich kommt auch hier die Bewegung zu einem Ende - jedoch in Form eines sehr kontrollierten Anhaltens, parallel mit von Rundstedts Zum-Punkt-Kommen, mit dem Abschließen seiner Rede. 

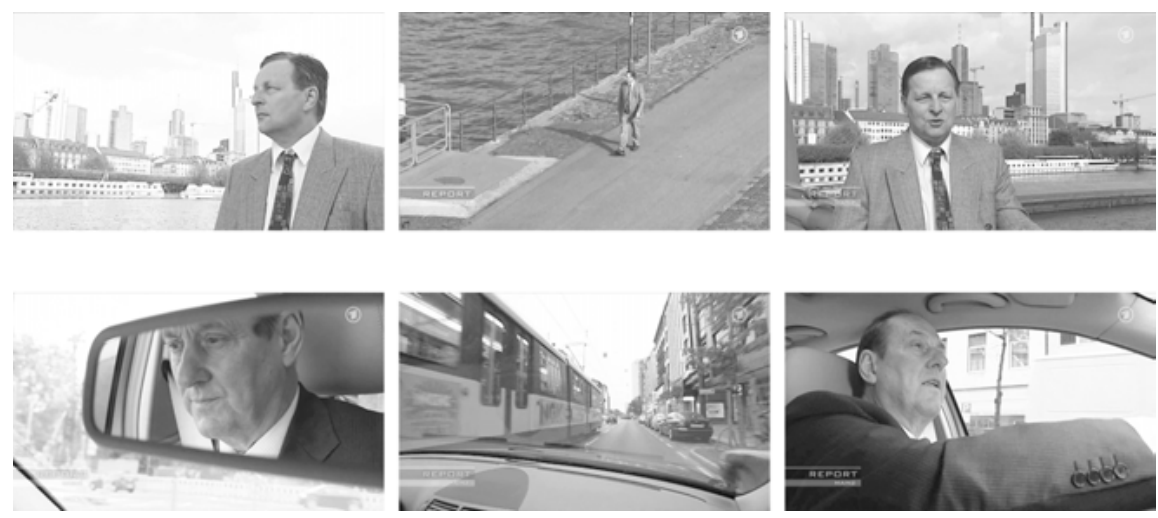

Abb. 40: Verlierer und Gewinner im zugespitzten Kontrast (REPORT MAINz-Beitrag zur Bankenkrise, dritte Szene; obere Reihe: erste Ausdrucksbewegungseinheit [02:16-02:54]; untere Reihe: zweite Ausdrucksbewegungseinheit [02:54-03:23]).

Sprachlich geht es jetzt jedoch weder um Gewinnen noch um Verlieren. Die Rede kommentiert vielmehr die Flüchtigkeit von Wut, Skandalen und Aufmerksamkeit.

Voice-over

„Aber: Die Wut derjenigen, die die Finanzkrise auszubaden haben, werde bald verraucht sein, glaubt Outplacement-Unternehmer Eberhard von Rundstedt.“

von Rundstedt

„Wir alle wissen, dass eben von Skandalen, über die das ganze Land spricht, nach ein, zwei Jahren nicht mehr die Rede ist. Das ist vor allen Dingen dann der Fall, wenn, und das passiert ja allzu oft, andere Skandale auftreten und die Öffentlichkeit sich anderen Problemen zuwendet.“

Mit der Ausdrucksbewegung entfaltet sich hierzu ein Bild von Gleichgültigkeit, das Gefühl eines das Außen ausschließenden Innens, das Gefühl einer geraden, ungestörten, kontinuierlichen Vorwärtsbewegung.

Die dritte Szene konfrontiert also einen Kleinanleger mit einem Berater - und dass hier von einer Konfrontation gesprochen werden kann, begründet sich eben noch allererst in der filmischen Expressivität und ihrer formalen Gestaltung: Durch sie realisiert sich eine Wahrnehmung von Verlierer und Gewinner als ein Aufeinander-Bezogensein, das zugleich ein Aufeinanderprallen und ein Sichdiametral-Gegenüberstehen beschreibt. ${ }^{10}$ So wiederholt das zweite Bewegungs-

10 Die beiden Ausdrucksbewegungen trennt eine Schwarzblende. Anders jedoch als beim Spielfilm, wo Schwarzblenden häufig Szenengrenzen markieren, ist die Schwarzblende an dieser Stelle dieses Beitrags noch Teil der Artikulation von Bewegung und Gegenbewegung, bei der auch die 
muster das erste in seinem formalen Montage-Rhythmus. Beide haben die gleiche Länge (etwa 30 Sekunden). Und beide sind eine Montage aus drei Einstellungen zwei kürzere mit Voice-over jeweils zu Beginn, gefolgt von einer längeren Originaltoneinstellung. Zugleich spiegeln die beiden Bewegungsmuster einander invertierend: Bildkomposition, Perspektive und Bewegung der Kamera (jeweils die Figurenbewegung parallelisierend) realisieren für den Kleinanleger eine Bewegung von rechts oben nach links unten, und für den Berater eine entgegengesetzte, nach rechts und vorwärts gerichtete Orientierung. Und auch die Höhepunkte beider Ausdrucksbewegungseinheiten - die entfernte Außenansicht des Kleinanlegers und die Subjektive des Beraters - verkehren einander in ihr Gegenteil. (Abb. 41)

Durch das Spiegelnde und Repetitive der beiden Bewegungsmuster zeigt diese Szene die Erfahrungen des Verlierer- und Gewinner-Seins in der Finanzkrise als unmittelbar und konstitutiv aufeinander bezogen. Und es passiert hier noch etwas anderes, das mit der grundsätzlichen Dynamik des Metaphorisierens zusammenhängt; damit, das verkörpertes Denken mit filmischen Bildern heißt, einen Ausdrucksparcours, ein Ganzes zu durchlaufen, einen Zusammenhang. Die Ausdrucksbewegung mit der Autofahrt evoziert eine Wahrnehmungserfahrung, die die Rede von der Flüchtigkeit von Wut, Skandalen und Aufmerksamkeit in Korrelation mit dem Gefühl einer geraden, fokussierten, ungestörten, kontinuierlichen Vorwärtsbewegung bringt. Es konstiuiert sich so mit und für die Berater eine Weltsicht, die von der Krise profitiert, ohne von der Situation der Krisenverlierer berührt, tangiert zu sein. Diese Perspektive schließt das Außen aus, das mit dem Krisengefühl behaftet ist, und bewegt sich unbehelligt durch dieses Außen hindurch. Zugleich aber vollziehen sich mit den Ausdrucksbewegungen, die die Kleinanleger zur Wahrnehmung bringen, im Sehen und Hören das Gefühl einer Verlusterfahrung und des Scheiterns. Das Nicht-Tangiert-Sein vom Verlieren ist also ein unmögliches Gefühl - das man dennoch im Zuschauen, im Wahrnehmungserleben, im Prozess des Metaphorisierens, im von den filmischen Bildern modellierten Prozess verkörperten Denkens realisiert. Eben aus solchen unauflösbaren Widersprüchen schöpft der Beitrag seine provozierende Energie, eben darüber beziehen diese filmischen Bilder gesellschaftspolitisch Stellung.

Sprache wirksam ist, wenn der Voice-over-Auftakt der zweiten Ausdrucksbewegung ein stark betontes „, $\underline{\text { ber: }}$ “ ist. 


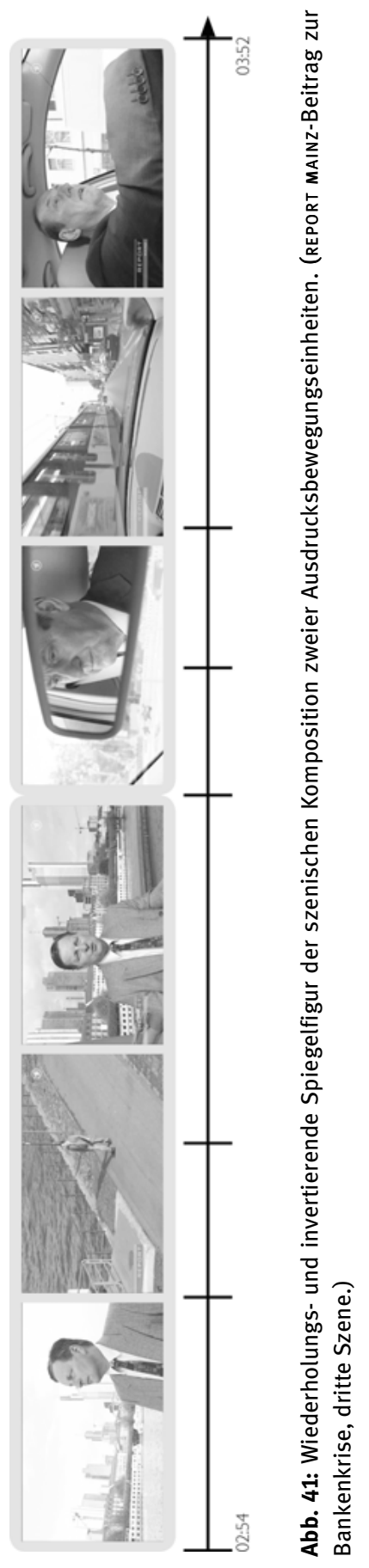




\section{Gewinnen verstehen}

Sechste Szene, 05:36-06:25

Im Zuge der bisherigen Betrachtungen einer Bedeutungskonstitution durch filmisches Metaphorisieren in einem journalistischen Fernsehformat wurden mit dem Polarisieren und dem Innehalten bereits zwei zeitliche Verlaufsformen angesprochen, mit denen die Aktivität des Metaphorisierens auf makroanalytischer Ebene selbst noch als eine Figuration gefasst werden kann. Zum Abschluss der Analyse ist nun noch kurz ein dritter solcher Aspekt anzusprechen: das pointierte Rahmen, wie es die letzte Szene des Beitrags realisiert (die zugleich auch Teil des Polarisierens ist).

Der Beitrag kehrt zurück zu den Beratern: Man kehrt zurück zur Qualität des geschlossenen Innens ebenso wie zum Oben - man kehrt zurück zu den Gewinnern der Bankenkrise (Abb. 42).

So sind hier in variierender wie auch wiederholender Weise Kreisformationen artikuliert: variierend, wenn zu Anfang eine Gesprächssituation von Rundstedts mit einem weiteren Berater durch eine Montage von Nah- und Großaufnahmen in Kombination mit einer langsamen, $180^{\circ}$-Kamerafahrt in einer Kreisbewegung inszeniert wird; und wiederholend, wenn gegen Ende wieder mit einer Teleobjektiv-Aufnahme der um einen runden Stehtisch gruppierten Berater eine geschlossene, auf ihr Inneres ausgerichtete Kreisformation bildkompositorisch gestaltet ist. Das Oben wiederum realisiert sich zum einen mit einer Großaufnahme von Rundstedts: Die Frage, ob die bereits gut laufenden Geschäfte demnächst noch besser laufen werden, bejaht der Geschäftsführer nicht nur verbal, sondern auch mit einer Kopfgeste: Sehr deutlich liegt die Betonung seines Nickens in der Aufwärtsbewegung. Zum anderen wird das Oben sprachlich eingeholt, wenn es zum Szenenanfang heißt:

Voice-over

„Diese Probleme werden die Manager ganz oben wohl nicht bekommen. Denn Top-Banker werden bei Eberhard von Rundstedt von ehemaligen Top-Bankern beraten.“

Mit diesem abschließenden Zurückkehren zu Ausdrucksqualitäten, mit denen der Beitrag in der ersten Szene Gewinnen inszeniert hatte, um dann in einem scharfen Kontrast hierzu das Verlieren $\mathrm{zu}$ entwickeln, vollzieht sich im Metaphorisieren eine Rahmungsfigur. Und dies geht einher mit einer Pointe. Denn hier wird nun der Ersetzungsprozess explizit, der der ersten Szene bereits implizit war, wenn dort im Zuge einer ironischen Distanzierung Outplacement als „optimales Instrument für gescheiterte Bankmanager“ beschrieben wurde, und parallel hierzu die geschlossene Kreisformation der feiernden Berater zu sehen war. Man sieht die gescheiterten Banker - die „nicht gesehen werden“ wollen, wie der Geschäfts- 
führer sagte - in den feiernden Beratern. Ein Verlieren steht so nicht mehr im scharfen Kontrast zum Verlieren: Die Realität, die sich im Sehen und Hören durch das vielfache Polarisieren gefestigt hat, ist nicht die Realität der Banker. Das weiche Fallen, von dem in der Anmoderation die Rede war, zeigt sich in dieser Erfahrungsform, die der Beitrag als Ganzes entfaltet.

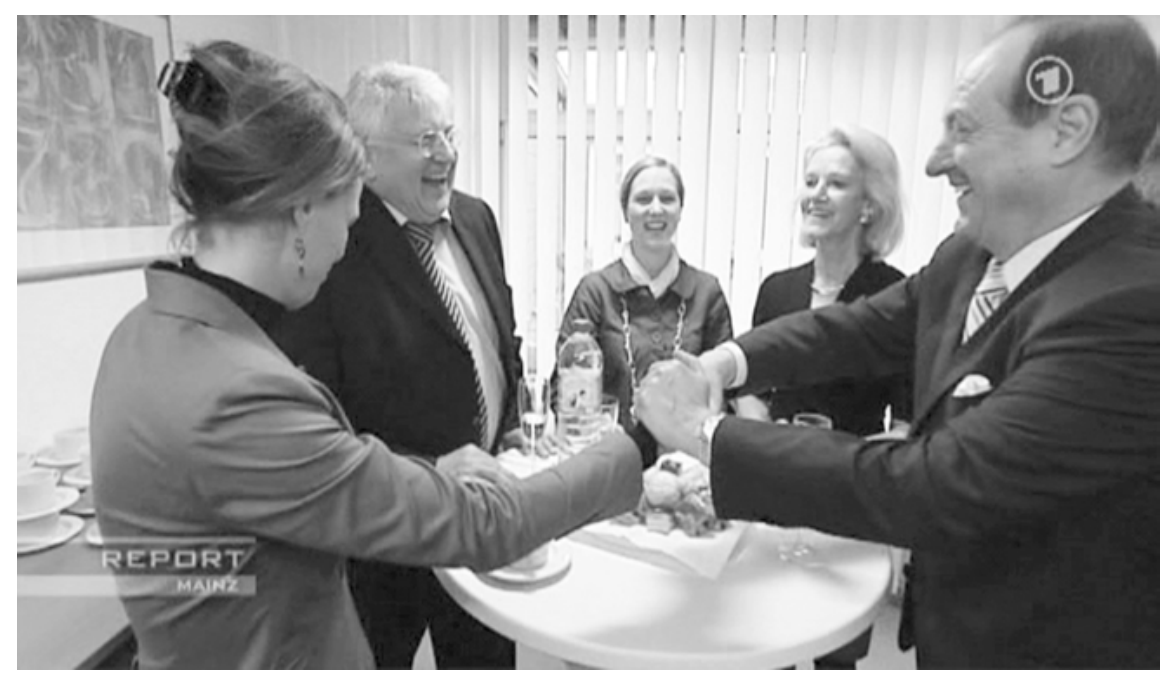

Abb. 42: Zum Oben und zum geschlossenen, auf ein Zentrum ausgerichteten Innen des Gewinnens zurückkehren und in den erfolgreichen Beratern die gescheiterten Bankmanager sehen - der Abschluss des REPORT MAINz-Beitrag zur Bankenkrise (sechste Szene, 05:36-06:25). 